\title{
Personal listening habits and the potential for hearing loss of Canadian university students
}

\author{
Christine Elizabeth Friesen and Andrew Papadopoulos \\ Department of Population Medicine, University of Guelph, Guelph, ON.
}

\begin{abstract}
Rationale: Personal and external factors, such as earphone type and music preference, can influence music volume adjustment beyond safe levels. The present study attempted to identify which factors are most influential on volume adjustment. Method: A cross-sectional survey of university students $(n=75)$ who use personal listening devices (PLD) was performed. Additionally, each participant's PLD music volume was measured through their earphones.

Results: On average, participants listened to music at safe ( $<85 \mathrm{~dB}$ ) but high levels $(79.8 \mathrm{~dB})$ for generally less than four hours per day. Nearly $60 \%$ of respondents used earbuds and half preferred "noisy" music genres such as hip-hop and rock/folk. The vast majority of respondents indicated listening to music while travelling by bus for the purpose of blocking out environmental noise or out of boredom. About $75 \%$ of the participants were categorized as "pro-noise". Most students claimed to respond to changing noise environments by adjusting music volume, but few enabled PLD built-in volume controls. Impact: This study determined that earphone type, listening environment, music genre, and listening duration were influential on an participants' adjustment of music volume. Further research is needed to assess earphone quality and to clearly elucidate more complex associations between external or personal factors and volume adjustment.
\end{abstract}

Key words: personal listening device, earphones, music genre, noise environment, listening duration.

\section{Introduction}

Technological advances since the 1970s have made listening to music a very personal and leisurely activity through the development of personal listening devices (PLDs). Media players have become compact with more storage space to permit long periods of listening. These improvements have led to an increased popularity of PLDs: an estimated $90 \%$ of university students own some form of PLD (Torre, 2008). Further, psychologists have speculated that young people turn to music as a way to escape reality or rebel against parental figures (Levey et al., 2011). Some even speculate that listening to the same music forms unspoken bonds between young people with similar tastes, though in a less direct manner as attending the same concert would (Levey et al., 2011).

The increased use of PLDs over the past few decades has led to concerns about the hearing health of young individuals who frequently listen to music, often at high volumes. Noiseinduced hearing loss (NIHL) is not a new phenomenon, as many studies have shown that workplace exposure to loud and prolonged noise can lead to temporary and permanent hearing loss (Levey et al., 2011). In 1998, the National Institute for Occupational Safety and Health of the United States issued regulations that permitted workers to be exposed to a

Corresponding author: Andrew Papadopoulos (email: apapadop@ uoguelph.ca) maximum sound pressure of 85 decibels $(\mathrm{dB})$ for no more than eight hours each day or forty hours per week. The Canadian Occupational Health and Safety Act (2014) lists similar recommendations for continuous noise exposure.

Recent research has been geared towards younger populations whose habits involve loud noise exposure during leisure and school activities. In 2008, it was reported that $17 \%$ of teenagers lost some of their hearing without being aware of their hearing loss (Rawool and Colligon-Wayne, 2008). High sound pressure directed close to the cochlea via headphones can lead to NIHL, which is currently second only to age as the most common cause of hearing loss. Hearing ability initially deteriorates at the higher range of frequencies (above $1 \mathrm{kHz}$ ), which is referred to as high-frequency hearing loss (HFHL; Berg and Serpanos, 2011). HFHL reduces one's ability to understand consonants, which is especially crucial in young adulthood where social activities and classroom learning are highly dependent upon the ability to communicate (Berg and Serpanos, 2011). Repeated loud noise exposure can even result in permanent, irreversible hearing loss (Vogel et al., 2007; Rawool and Colligon-Wayne, 2008; Berg and Serpanos, 2011; Levey et al., 2011).

\section{Listening habits}

Conflicting evidence exists regarding causes of noise-induced hearing loss, indicating that there may be a combination of 
factors at work. Levey et al. (2011) determined that volume and duration of exposure both contributed to hearing loss; more than half of study participants at a New York university reported noise-related hearing damage in relation to their listening habits, which consisted of listening to music at a volume greater than the recommended $85 \mathrm{~dB}$ for 18.4 hours per week on average. On the other hand, several other studies supported either volume or duration as the sole cause of hearing loss (Rawool and Colligon-Wayne, 2008; Zocoli et al., 2009; Berg and Serpanos, 2011; Tung and Chao, 2013). Therefore, it is still unclear whether only a few or repeated loud-noise exposures are needed to produce hearing loss.

\section{Effects of PLD use}

Reported symptoms of ear damage were consistent among various study populations, but the severity of the problem varied among participants. Studies that involved strongly homogenous populations reported higher prevalence of temporary tinnitus (i.e., ringing in the ears for more than 24 hours in the absence of sound) than studies involving heterogeneous groups, making it difficult to compare their respective statistics. Homogeneous populations, such as a small private Brazilian high school and an all-female foster residence, reported that $69 \%$ and $99.7 \%$ of regular PLD users had tinnitus, respectively (Zocoli et al., 2009; Berg and Serpanos, 2011). However, two heterogeneous populations reported tinnitus $21.6 \%$ to $26.2 \%$ of the time in relation to PLD use (Widén and Erlandsson, 2004; Marcoux et al., 2012), whereas a third study involving American college students found that $66 \%$ of participants reported tinnitus after leisure noise exposure (Rawool and Colligon-Wayne, 2008). The studies using heterogeneous groups (Widén and Erlandsson, 2004; Marcoux et al., 2012) provide generalizable statistics, whereas the homogenous population-based models (Zocoli et al., 2009; Berg and Serpanos, 2011) would be useful for determining gender and socio-economic differences in correlation with PLD use and preventative attitudes.

\section{Attitudes and awareness}

Despite general awareness of the risks of loud noise exposure, the number of individuals who have reported not using hearing protection or taking preventative measures is alarming. Fortytwo percent of American college students were aware of the consequences of loud noise exposure and $85 \%$ were aware that hearing loss is irreversible (Crandell et al., 2004; Rawool and Colligon-Wayne, 2008). Contrary to their awareness, $68 \%$ of American college students purposely listened to very loud music or sat next to speakers at concerts (Rawool and Colligon-Wayne, 2008). Similarly, participants from the Brazilian private high school were generally anti-noise when compared with American and Swedish students, although these attitudes did not seem to prompt risk avoidance from these groups (Zocoli et al., 2009).

Hearing protection was generally understood to be important in loud noise situations, yet it was rarely worn by participants at social events owing to a perceived decrease in pleasurable listening experience or from negative social stigma
(Crandell et al., 2004; Chung et al., 2005; Rawool and Colligon-Wayne, 2008; Zocoli et al., 2009). Young adults tended to consider symptoms of hearing loss as minor when compared with other issues such as STDs, drug abuse, and depression (Chung et al., 2005). It has also been suggested that younger teenagers are unaware or apathetic towards the risks and thus do not take these actions when in college (Widén and Erlandsson, 2004). Gender differences and socio-economic status may also affect an individual's listening habits and risktaking behaviour.

The current study sought to determine how external and personal factors, such as listening environment or music preference, might influence an individual's adjustment of music volume when using a PLD. Additionally, this study sought to elucidate young adults' attitudes towards loud music and awareness of the associated risks.

\section{Methods}

\section{Participants}

Seventy-five University of Guelph students (32 males, 42 females) mainly between the ages of 17-22 (14 participants were over the age of 23), were recruited over the course of three days as they passed the research booth located at two popular areas on campus. Participants were eligible for the study if they were PLD users and provided informed consent. No efforts were taken to determine hearing ability of participants prior to sound level measurement. This study obtained ethics approval from the University.

\section{Procedure and measures}

Participants were invited to have their earphone music levels measured and were asked to complete a survey requesting information regarding: demographics (i.e. age, gender, year of study), earphone type, music genre preference, self-reported duration of listening, reasons for listening to music, and attitudes toward noise (see Appendices 1 and 2 for a list of the survey items).

Music volume as emitted from the participants' earphones was determined using a $3 \mathrm{M}$ SoundPro DL $1 / 1$ and $1 / 3$ Octave Datalogging RTA Sound Level Meter with a Class 2 microphone. The SoundPro Meter was pre-calibrated and set to measure sound pressure level using the following criteria: slow response time, $3 \mathrm{~dB}$ exchange rate, $85 \mathrm{~dB}$ criterion level, and $140 \mathrm{~dB}$ upper limit. Sound was measured using A-weighted frequency to mimic the human ear's response to noise. The levels measured from participants' earphones were assumed representative of their typical listening levels.

Two different methods were used to accommodate for different earphone types. Earbuds and insert earphones were placed inside a hard plastic adaptor that fit over the sound meter's microphone to minimize the effect of background noise on the measurement. A windscreen was attached to the microphone for supra-aural and circumaural headphones, against which the headpieces were firmly placed; the researcher 
also cupped a hand over the apparatus to minimize background noise. Environmental noise was measured at each location on separate data collection days; although there was a large difference between noise environments (ranging from $54.6 \mathrm{~dB}$ to $73.1 \mathrm{~dB}$ ), this did not seem to affect recorded music volumes. Statistical significance $(p<0.05)$ was determined with a two sample $t$ test assuming unequal variances. Calculations were performed using Microsoft Excel 2010.

Upon completion of the survey and volume measurement, participants were informed verbally and in written form about existing noise level recommendations to increase their awareness about the risk of noise-induced hearing loss from loud music exposure. This information was based on recommendations of the Canadian Centre for Occupational Health and Safety (CCOHS, 2014) in Canada.

\section{Results}

\section{Mean PLD volume}

At the time of data collection, the average participant was listening to their PLD at $79.8 \mathrm{~dB}$ (Figure 1). Approximately $30 \%$ of participants (or 22 individuals) listened to music at or above $85 \mathrm{~dB}$ at the time of data collection and were considered "high-risk" for noise-induced hearing loss.

\section{Factors affecting volume}

Appendix 1 displays the questions and response rates for eight questions regarding personal preference, external factors, and demographic information that were hypothesized to have an effect on volume adjustment.

\section{Earphone type}

A comparison of four types of earphones determined that $57.3 \%$ of participants listened to their PLD using non-noise cancelling earbuds, which was associated with the highest music

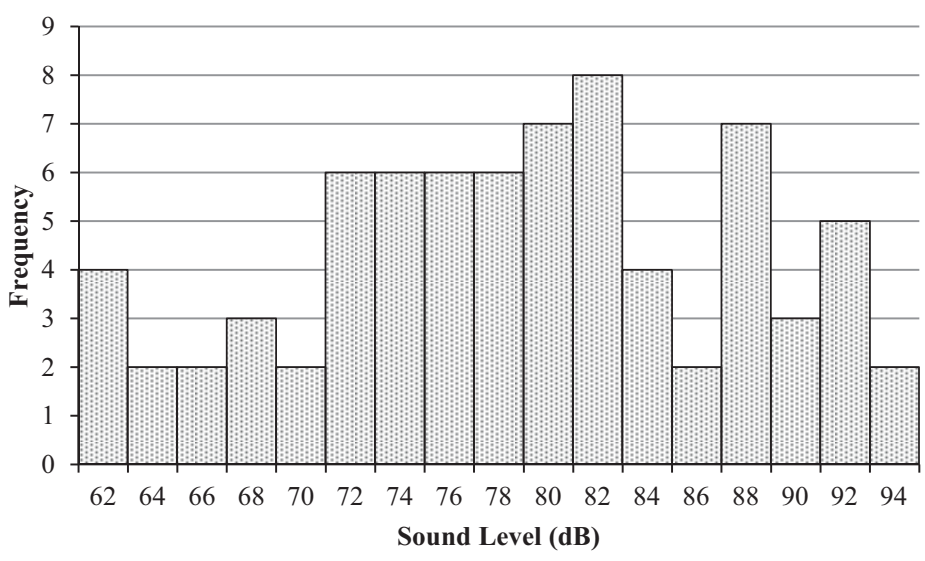

Fig. 1. Histogram of measured music volumes $(n=75$, mean $=79.812$ and SD $=8.548$ ). The histogram displays the frequency of measured music volumes within a range of $2 \mathrm{~dB}$ (median $=80.1 \mathrm{~dB}$ and range $=62.7-95.6 \mathrm{~dB}$ ). volume (81.2 dB; see Figure 2). Participants who listened to music above the recommended $85 \mathrm{~dB}$ (high-risk individuals) reported using earbuds $72.7 \%$ of the time, compared with $50.9 \%$ of those who listened below $85 \mathrm{~dB}$ (low-risk individuals).

\section{Preferred music genre}

The majority of participants selected hip-hop (52\%) and rock/ folk music (50.7\%; see Figure 3) as their preferred music genres. Participants who reported listening to electronic music (28.0\%) listened to their music significantly louder $(82.4 \mathrm{~dB}, p=$

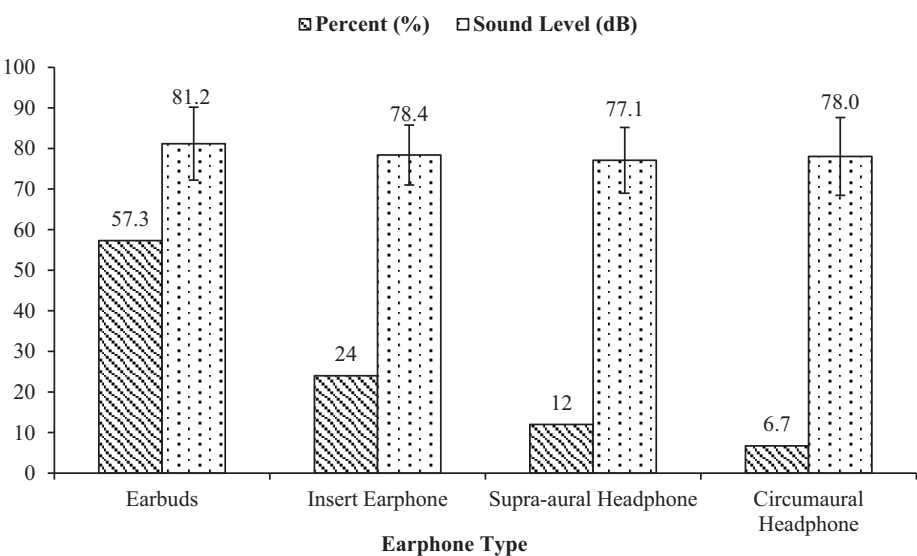

Fig. 2. Mean sound level (in $\mathrm{dB}, \pm 1 \mathrm{SD}$ ) and reported use of four earphone types (earbuds, insert earphone, supraaural headphone, and circumaural headphone). Numerical values over bars indicate percentage (for dashed bars) and sound level (for dotted bars).

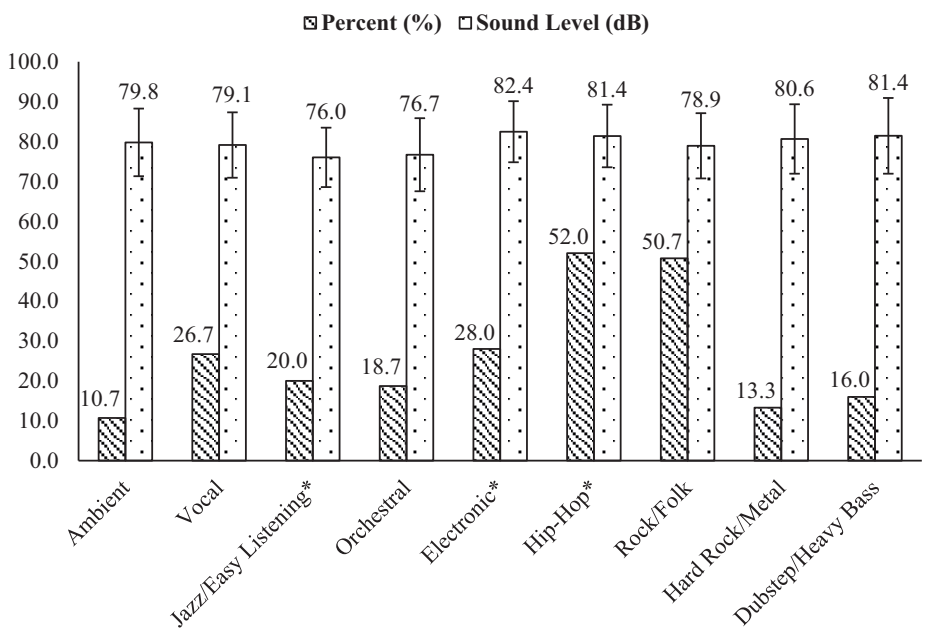

Fig. 3. Mean listening volume (in $\mathrm{dB}, \pm 1 \mathrm{SD}$ ) and preferred music genre (\%). Significant differences $(p<$ 0.05 ) in sound level between jazz/easy listening and electronic music genres, and between jazz/easy listening and hip-hop music genres is indicated by asterisks $(*)$. Numerical values over the bars indicate percentage (for dashed bars) and sound level (for dotted bars). 
$0.0169)$ than listeners of jazz or easy listening genres $(20.0 \%$, $76.0 \mathrm{~dB}$ ). Further, listening to hip-hop was associated with a significantly louder listening volume $(81.4 \mathrm{~dB}, p=0.0276)$ than listening to jazz or easy listening music genres. However, there was no noticeable difference between preferred music genres when comparing high-risk with low-risk individuals.

\section{Listening duration (Daily)}

Participants generally reported listening to music for any duration less than 4 hours per day. Few participants reported listening for more than 5 hours per day (16.7\%). Listening to music for more than 7 hours per day was reported by $6.7 \%$ of participants and was found to be associated with a significantly lower music volume $(69.9 \mathrm{~dB}, p=0.0449)$ than the other four listening duration categories; no participants in the $7+$ hours/ day group listened to music $>85 \mathrm{~dB}$ and thus were all considered low-risk for this category.

\section{Listening environment}

Eighty-four percent of participants reported listening to music while travelling by bus, compared with a somewhat even distribution among the other choices (48.0-57.3\%; see Figure 4). Reported sound levels were highest when participants indicated they listened to music on the bus $(80.0 \mathrm{~dB})$ and on the first floor of the library (or somewhere with similar noise levels; $79.5 \mathrm{~dB}$ ). The majority of both high-risk and low-risk participants listened to music on the bus. Although not statistically significant, PLDs were used more often in quieter environments by low-risk individuals than high-risk individuals $(31.8 \%-40.9 \%$ and $54.7 \%-66.0 \%$, respectively).

\section{Reasons for listening to music}

Participants selected up to six options regarding their reasons for listening to music. The majority of participants listened to music to block out the environment (including background

๒ Percent (\%) $\square$ Sound Level (dB)

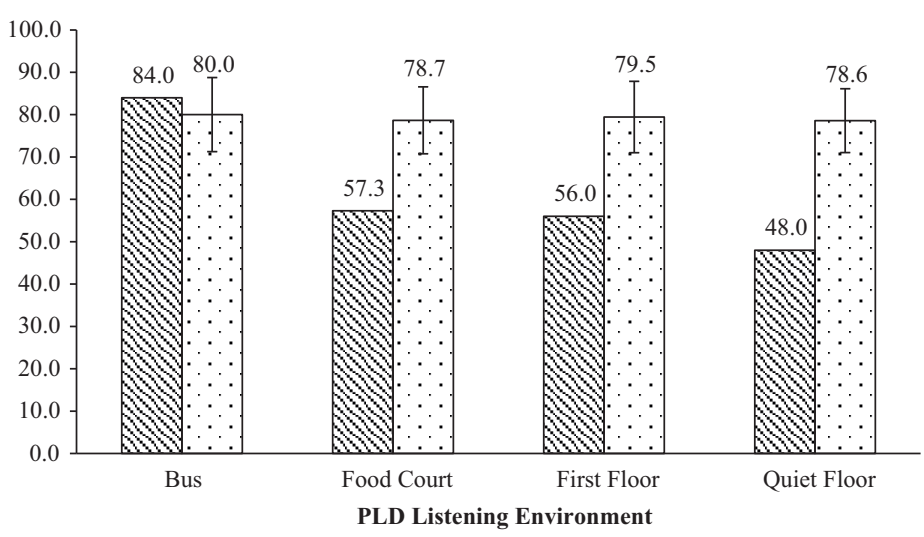

Fig. 4. Mean listening volume (in $\mathrm{dB}, \pm 1 \mathrm{SD}$ ) and typical noise environment for listening to music (\%). Numerical values over bars indicate percentage (for dashed bars) and sound level (for dotted bars). noise and other people), to gain inspiration, or to simply pass time (67.6-68.9\%). Far fewer high-risk participants (27.3\%) indicated using music to study compared with $60.4 \%$ of lowrisk participants. The highest mean recorded sound level was associated with performing leisurely activities $(80.9 \mathrm{~dB})$. Music levels were lowest for those who listened to music while playing video games $(76 \mathrm{~dB})$, although the importance of this value is questionable due to the low response rate for this option.

\section{Experience with tinnitus}

Seventy-seven percent of participants reported never experiencing tinnitus when exposed to noise compared with a small $16.2 \%$ of participants who reported experiencing tinnitus for less than 1 hour. An even smaller group (2 participants) experienced tinnitus for 1-12 hours after noise exposure, and these individuals had a lower listening volume $(72.7 \mathrm{~dB})$ than all other categories. Tinnitus lasting less than one hour was associated with the highest music volume $(84.1 \mathrm{~dB})$. Student's $t$ test was not used to analyze data from responses more than 1 hour in length since there was a low response rate for each option.

\section{Age and gender}

The majority (81.3\%) of participants were aged 17-22 years, the typical age for undergraduate students, and distributed roughly equal between the genders ( $43.2 \%$ males and $56.8 \%$ females). Age and gender do not seem to have a significant effect on volume adjustment.

\section{Year of study}

Nearly half $(41.3 \%)$ of the participants were fourth year undergraduate students, with a generally even spread of participants across the first-year through third-year students (14.7-20.0\%). First years $(83.4 \mathrm{~dB})$ listened to music significantly louder than third-year students $(77.0 \mathrm{~dB} ; p=$ 0.0416); however, this relationship did not continue into older years (fourth year undergraduates and post-graduates) indicating some error due to an unequal distribution of participants across year of study categories.

\section{Attitudes and awareness}

Appendix 2 displays the statements and response rates for 19 statements regarding attitudes toward noise and knowledge about ear safety, with a focus on external factors (i.e., traffic noise or understanding speech in meetings). Statements were rated from one (strongly agree) to five (strongly disagree) and scores were totaled to categorize participants more "anti-noise" or "pro-noise" (i.e., noise is not regarded as a problem). Seventy percent of participants were pro-noise, although $53.4 \%$ preferred quieter environments in general. Despite clear differences in attitudes between participants, no significant difference was found between listening levels for the two groups (anti-noise $=80.2 \mathrm{~dB}$, pro-noise $=79.7 \mathrm{~dB}$ ). 


\section{Discussion}

Safe noise exposure recommendations according to NIOSH (1998) and CCOHS (2014) are based on research performed in the workplace, in which individuals were exposed to noise during an 8-12 hour shift. Therefore, these agencies recommend a maximum noise exposure of $85 \mathrm{~dB}$ for eight hours per day or 40 hours per week; this changes to one hour maximum exposure if the sound exceeds $90 \mathrm{~dB}$ (CCOHS, 2014). These recommendations, although useful for determining a safe occupational listening level, are not applicable to the average music listener who is likely not exposed to loud music for extended periods as indicated by NIOSH and CCOHS. Some researchers, such as Fligor and Cox (2004), suggest headphone use should be limited to one hour of use or less per day at a maximum volume setting of $60 \%$ when using supra-aural headphones. The current study determined many university students were not in this sort of habit.

It is evident from the present study that almost one-third of participants listened to music above the recommended $85 \mathrm{~dB}$. Students seemed to generally use earbuds (not noisecancelling), listened to music loudly to compensate for environmental noise, preferred "noisier" music genres and ignored built-in iPod volume controls. Participants were generally aware of the risk of loud noise exposure; however, this knowledge may not have been applied to certain aspects of music listening that have been shown to be associated with an increase in volume.

\section{Music genre}

Music genre had an effect on an individual's adjustment of music volume. Jazz music, (like ambient, vocal, orchestral and rock/folk genres), generally involves softer tones with a focus on vocals along with a solo instrument. Jazz music is a "quieter" genre and may be associated with leisurely activities that do not require a high volume for motivation. Electronic and hip-hop music, on the other hand, is generally repetitive and fast-paced with many sounds and noises incorporated into the tune. It is likely that "noisier" electronic and hip-hop music (as well as hard rock/metal and dubstep/heavy bass) is associated with high-energy tasks in a potentially loud environment (such as a short-term study session, walking across campus or a break between classes). This does not necessarily indicate that electronic or hip-hop music listeners are at higher risk for hearing loss; it is possible that participants selected a combination of "loud" and "soft" music when completing the survey.

\section{Listening duration}

Listening to music for more than seven hours per day was significantly associated with a lower music volume than any duration shorter than seven hours per day. This suggests that participants may engage in certain activities up to six hours in length that cause them to increase the volume (such as riding the bus, walking to class, or studying during a break). These are instances when music volume was higher during high-paced, loud environments for a relatively short period. Participants who reported listening to music for more than seven hours per day may spend most of that time studying in an environment free of distractions (like a quiet floor of a library), engaging in leisurely activities, or exercising. These individuals likely listen to music for a longer duration in one setting, rather than at short intervals. This suggests that longer listening durations could be safer than short durations when the short interval induces a higher volume, contrary to findings by Tung and Chao (2013) and Levey et al. (2011).

Despite listening to music close to $85 \mathrm{~dB}$, only a few participants reported experiencing tinnitus. This result contrasts with the work of other researchers worldwide (Chung et al., 2005; Rawool and Colligon-Wayne, 2008; Zocoli et al., 2009; Berg and Serpanos, 2011; Tung and Chao, 2013). Unlike Widén and Erlandsson (2004) and Rawool and Colligon-Wayne (2008), the present study did not assess any other symptoms of ear damage, such as ear pain or irritation, and based overall ear health on self-reported survey items; assessment of hearing ability was beyond the scope of this study.

\section{Conclusion}

It is evident that university students are aware that loud music can be dangerous; however, they are less aware about how earphone quality/type, music genre, listening environment, and listening duration can affect volume adjustment. Participants mainly used non-noise cancelling earbuds to listen to music at $79.8 \mathrm{~dB}$ on average, which is considered safe but close to the maximum recommended level of $85 \mathrm{~dB}$. Music was generally listened to while traveling by bus, which is a high noise environment. Additionally, participants preferred noisier electronic and hip-hop music to quieter jazz music, which may be influenced by listening environment or reason for listening. Participants who listened to music for more than seven hours per day tended to listen to music significantly quieter than listeners for any duration less than seven hours, suggesting an association with listening environment, reasons for listening and music preference. This study also determined that having an option to enable a built-in volume control is not enough to prevent potentially damaging loud noise exposure, since many PLD users disregard this option. However, few participants reported tinnitus and individuals tended to reduce music volume when transitioning from louder to quieter environments.

Future research endeavors should be tailored towards improving earphone quality and developing guidelines more specific to earphone and PLD users. Further research also needs to address the effects of environmental and personal factors on volume adjustment to establish more conclusive associations than in the present study. Upon establishing a database, information on this topic could then be used for educational purposes to promote safe PLD listening behaviours.

\section{References}

Berg, A.L., and Serpanos, Y.C. 2011. High frequency hearing sensitivity in adolescent females of a lower socioeconomic status 
over a period of 24 years (1985-2008). J. Adoles. Health, 48: $203-$ 208. PMID: 21257121. doi: 10.1016/j.jadohealth.2010.06.014.

Canadian Centre for Occupational Health \& Safety (CCOHS). 2014. Noise: Occupational Exposure Limits in Canada. Available at: http://www.ccohs.ca/oshanswers/phys_agents/exposure_ can.html.

Chung, J.H., Des Roches, C.M., Meunier, J., and Eavey, R.D. 2005. Evaluation of noise-induced hearing loss in young people using a web-based survey technique. Pediatrics, 115: 861-867. PMID: 15805356. doi: 10.1542/peds.2004-0173.

Crandell, C., Mills, T.L., and Gauthier, R. 2004. Knowledge, behaviors, and attitudes about hearing loss and hearing protection among racial/ethnically diverse young adults. J. Natl. Med. Assoc., 96: 176-186. PMID: 14977276.

Fligor, B.J., and Cox, L.C. 2004. Output levels of commercially available portable compact disc players and the potential risk to hearing. Ear Hear., 25(6): 513-527. PMID: 15604913. doi: 10.1097/00003446-200412000-00001.

Levey, S., Levey, T., and Fligor, B.J. 2011. Noise exposure estimates of urban MP3 player users. J. Speech Lang. Hear. Res., 54: 263-277. PMID: 20689033. doi: 10.1044/1092-4388(2010/09-0283).

Marcoux, A.M., Feder, K., Keith, S.E., Marro, L., James, M.E., and Michaud, D.S. 2012. Audiometric thresholds among a Canadian sample of 10 to 17 year old students. J. Acoust. Soc. Am., 131: 2787-2798. PMID: 22501057. doi: 10.1121/1.3689550.

Rawool, V.W., and Colligon-Wayne, L.A. 2008. Auditory lifestyles and beliefs related to hearing loss among college students in the USA. Noise Health, 10: 1-10. PMID: 18270402.

Torre, P. 2008. Young adults' use and output level settings of personal music systems. Ear Hear., 29: 791-799. PMID: 18633323. doi: 10.1097/AUD.0b013e31817e7409.

Tung, C.-Y., and Chao, K.-P. 2013. Effect of recreational noise exposure on hearing impairment among teenage students. Res. Dev. Disabil., 34: 126-132. PMID: 22940166. doi: 10.1016/ j.ridd.2012.07.015.

Vogel, I., Brug, J., van der Ploeg, C.P.B., and Raat, H. 2007. Young people's exposure to loud music: A summary of the literature. Am. J. Prev. Med., 33: 124-133. PMID: 17673100. doi: 10.1016/ j.amepre.2007.03.016.

Widén, S.E.O., and Erlandsson, S.I. 2004. Self-reported tinnitus and noise sensitivity among adolescents in Sweden. Noise Health, 7: 29-40. PMID: 15703147.

Zocoli, A.M.F., Morata, T.C., Marques, J.M., and Corteletti, L.J. 2009. Brazilian young adults and noise: Attitudes, habits, and audiological characteristics. Int. J. Audiol., 48: 692-699. PMID: 19863355. doi: 10.1080/14992020902971331.

\section{Appendix 1: Responses to survey items regarding listening habits and demographic information.}

\section{Question}

1. What type of earphones do you use?

Earbuds

Insert earphone

Supra-aural headphone

Circumaural headphone

2. What types of music do you mainly listen to? (Choose up to 3 ).

\begin{tabular}{|l|r|}
\hline Ambient & $10.7(8)$ \\
\hline Vocal & $26.7(20)$ \\
\hline Jazz/Easy listening & $20.0(15)$ \\
\hline Orchestral & $18.7(14)$ \\
\hline Electronic & $28.0(21)$ \\
\hline Hip-hop & $52.0(39)$ \\
\hline Rock/Folk & $50.7(28)$ \\
\hline Hard Rock/Metal & $13.3(10)$ \\
\hline Dubstep/Heavy bass & $16.0(12)$ \\
\hline
\end{tabular}


Appendix 1 (continued).

\begin{tabular}{|l|l|}
\hline Question & \% (no.) \\
\hline 3. How many hours per day do you listen to music using earphones? & $24.0(18)$ \\
\hline Less than one hour & $30.7(23)$ \\
\hline $1-2$ hours & $28.0(21)$ \\
\hline $3-4$ hours & $10.7(8)$ \\
\hline $5-6$ hours & $6.7(5)$ \\
\hline $7+$ hours & $(5)$
\end{tabular}

4. In what type(s) of noise environment(s) do you usually listen to music using earphones? (Check all that apply).

Traveling by bus

University Centre food court

$84.0(63)$

First floor of the library

$57.3(43)$

A quiet floor of the library

5. For what reasons(s) do you listen to music using headphones? (Check all that apply).

To concentrate while studying

$52.7(39)$

To keep motivated while playing video games

$14.9(11)$

While performing other leisure activities (i.e. reading, knitting, drawing)

$52.7(39)$

To block out environmental noise

$68.9(51)$

To block out thoughts or people, to 'escape from reality' or to gain inspiration

$68.9(51)$

Out of boredom

$67.6(50)$

6. Do you experience tinnitus after exposure to noise? If so, how long does it last?

I do not experience tinnitus.

$77.0(57)$

Less than one hour

$16.2(12)$

1-12 hours

$2.7(2)$

One day

$1.4(1)$

Several days

$1.4(1)$

I am always experiencing tinnitus.

7. Age

$17-20$

34.7 (26)

$20-22$

46.7 (35)

$23+$

$18.7(14)$

8. Gender

Male

$43.2(32)$

Female 
Appendix 1 (continued).

\begin{tabular}{|l|c|}
\hline Question & $\%$ (no.) \\
\hline 9. Year of Study & $20.0(15)$ \\
\hline 1st year undergraduate & $14.7(11)$ \\
\hline 2nd year undergraduate & $17.3(13)$ \\
\hline 3rd year undergraduate & $41.3(31)$ \\
\hline 4th year undergraduate & $6.7(5)$ \\
\hline Other & \\
\hline
\end{tabular}

Appendix 2. Responses to survey items regarding attitudes and education ( $1=$ Strongly Agree; $5=$ Strongly Disagree).

\begin{tabular}{|c|c|c|c|c|c|}
\hline & 1 & 2 & 3 & 4 & 5 \\
\hline & $\%$ (no.) & $\%$ (no.) & $\%$ (no.) & $\%$ (no.) & $\%$ (no.) \\
\hline I usually complain about having turned the TV volume too high. & $13.3(10)$ & $24.0(18)$ & $32.0(24)$ & $20.0(15)$ & $10.7(8)$ \\
\hline I cannot hear the doorbell ring or telephone ring. & $9.3(7)$ & $5.3(4)$ & $6.7(5)$ & $17.3(13)$ & $61.3(46)$ \\
\hline I am unable to hear calls from a distant room. & $6.7(5)$ & $6.7(5)$ & $13.3(10)$ & $30.7(23)$ & $42.7(32)$ \\
\hline I am unable to talk to others on noisy roadsides. & $5.4(4)$ & $13.5(10)$ & $24.3(18)$ & $29.7(22)$ & $27.0(20)$ \\
\hline $\begin{array}{l}\text { It is more difficult for me than for people in general to hear what } \\
\text { others say in noisy environments. }\end{array}$ & $4.0(3)$ & $24.0(18)$ & $18.7(14)$ & $22.7(17)$ & $30.7(23)$ \\
\hline I usually need to ask others to repeat themselves. & $2.7(2)$ & $26.7(20)$ & $20.0(15)$ & $29.3(22)$ & $21.3(16)$ \\
\hline $\begin{array}{l}\text { I have misunderstandings in meetings or discussions because I cannot } \\
\text { hear others clearly. }\end{array}$ & $4.0(3)$ & $10.7(8)$ & $20.0(15)$ & $30.7(23)$ & $34.7(26)$ \\
\hline I need to sit in the front of a classroom to hear the speaker clearly. & $8.0(6)$ & $12.0(9)$ & $13.3(10)$ & $34.7(26)$ & $32.0(24)$ \\
\hline $\begin{array}{l}\text { I often consider stopping certain activities if my surroundings become } \\
\text { too loud. }\end{array}$ & $9.3(7)$ & $33.3(25)$ & $24.0(18)$ & $20.0(15)$ & $13.3(10)$ \\
\hline $\begin{array}{l}\text { If not listening to music, I require earplugs during leisure activities or } \\
\text { while studying. }\end{array}$ & $10.7(8)$ & $12.0(9)$ & $14.7(11)$ & $28.0(21)$ & $34.7(26)$ \\
\hline I prefer quiet environments in general. & $22.7(17)$ & $30.7(23)$ & $33.3(25)$ & $4.0(3)$ & $9.3(7)$ \\
\hline I think classrooms should be calm and quiet. & $29.3(22)$ & $32.0(24)$ & $24.0(18)$ & $6.7(5)$ & $8.0(6)$ \\
\hline I find traffic noise to be disturbing and hard to ignore. & $9.3(7)$ & $25.3(19)$ & $32.0(24)$ & $24.0(18)$ & $9.3(7)$ \\
\hline I find the noise while travelling by bus to be uncomfortably loud. & $6.7(5)$ & $20.0(15)$ & $20.0(15)$ & $36.0(27)$ & $17.3(13)$ \\
\hline I find that sounds from appliances are uncomfortably loud. & $6.7(5)$ & $26.7(20)$ & $24.0(18)$ & $28.0(21)$ & $14.7(11)$ \\
\hline $\begin{array}{l}\text { I find that the sound levels in the University Centre are too loud for } \\
\text { comfort. }\end{array}$ & $5.3(4)$ & $17.3(13)$ & $20.0(15)$ & $44.0(33)$ & $13.3(10)$ \\
\hline I feel powerless when I cannot escape loud sounds. & $12.0(9)$ & $24.0(18)$ & $17.3(13)$ & $18.7(14)$ & $28.0(21)$ \\
\hline $\begin{array}{l}\text { I tend to lower music volume when transitioning to a quieter } \\
\text { environment. }\end{array}$ & $42.7(32)$ & $29.3(22)$ & $8.0(6)$ & $12.0(9)$ & $8.0(6)$ \\
\hline $\begin{array}{l}\text { I enable my iPod's built-in volume control that prevents the volume to } \\
\text { exceed a certain level. }\end{array}$ & $14.9(11)$ & $17.6(13)$ & $14.9(11)$ & $21.6(16)$ & $31.1(23)$ \\
\hline
\end{tabular}

